calendar period of employment, and attained age at risk. Poisson regression was used to model the risk (RR) of incident leukemia at increasing levels of cumulative gamma radiation exposure, adjusting for calendar period and attained age.

Results Between 1969 and 2005, 116 incident cases of leukemia were identified. On average, these miners were employed for 4.4 years with a mean cumulative dose of 5.25 millisieverts $(\mathrm{mSv})$. With exposure lagged by 2 years, preliminary analyses showed that when compared to the referent group $(0 \mathrm{mSv})$, those with $>30 \mathrm{mSv}$ of cumulative gamma dose had a non-statistically significant increase in the risk of leukemia diagnosis ( $R R=2.04,95 \% \mathrm{CI}: 0.93,4.51)$ with increasing, linear trend $(\mathrm{p}=0.08)$.

Conclusions Although our results did not show a statistically significant relationship between gamma radiation and leukemia incidence, it is likely due to low statistically power. Future work may include pooling the Ontario Uranium Miners cohort with other similar cohorts to better quantify the potential associated risks.

\section{O6B.4 LARYNGEAL CANCER RISKS IN WORKERS EXPOSED TO LUNG CARCINOGENS: EXPOSURE-EFFECT ANALYSES USING A QUANTITATIVE JOB EXPOSURE MATRIX}

\begin{abstract}
${ }^{1}$ Amy Hall*${ }^{*}{ }^{2}$ Hans Kromhout, ${ }^{1}$ Joachim Schüz, ${ }^{2}$ Lützen Portengen, ${ }^{2}$ Susan Peters, ${ }^{2}$ Roel Vermeulen, ${ }^{3,7}$ Paolo Boffetta, ${ }^{1,7}$ Paul Brennan, ${ }^{4,7}$ Danièle Luce, ${ }^{4,7}$ Isabelle Stücker, ${ }^{5,7}$ Heribert Ramroth, ${ }^{6,7}$ Amy Lee Yuan-Chin, ${ }^{6,7}$ Mia Hashibe, ${ }^{1}$ Kurt Straif, ${ }^{1}$ Ann Olsson. ${ }^{1}$ International Agency for Research on Cancer, Lyon, France; ${ }^{2}$ Institute for Risk Assessment Sciences, Utrecht University, Utrecht, The Netherlands; ${ }^{3}$ /cahn School of Medicine at Mount Sinai, New York, USA; ${ }^{4}$ Institut National de la Santé et de la Recherche Médicale, Paris, France; ${ }^{5}$ University of Heidelberg, Heidelberg, Germany; ${ }^{6}$ University of Utah, Salt Lake City, USA; ${ }^{7}$ on behalf of the International Head and Neck Cancer Epidemiology Consortium
\end{abstract}

\subsection{6/OEM-2019-EPI.145}

Background Various occupational agents are suspected risk factors for laryngeal cancer. However, individual studies are often inadequate to investigate how such exposures relate to this relatively rare outcome. Important limitations include small sample sizes, lack of detail on exposure, and inadequate adjustment for confounders.

Methods This study applied quantitative estimates via SYNJEM for four established occupational lung carcinogens to case-control data from five (Western European and Latin American) studies within the International Head and Neck Cancer Epidemiology Consortium. Participant job histories for $\mathrm{n}=2256$ laryngeal cancer cases and $\mathrm{n}=7857$ controls recruited from 1989-2007 were linked with quantitative SYN-JEM estimates via ISCO-68 codes. Logistic regression models were applied to assess the effects of occupational exposure to asbestos, respirable crystalline silica (RCS), chromium-VI, and nickel on laryngeal cancer risk while controlling for tobacco and alcohol consumption.

Results Increased ORs were observed for all agents evaluated. In males, a positive exposure-effect relationship was observed with duration of RCS exposure (p-value for trend <0.01). Relatively strong effects were also observed in the highest categories of career-cumulative chromium-VI exposure (>90\%ile: $\mathrm{OR}=2.21,95 \% \mathrm{CI}=1.13-4.33)$ and career-average exposure (>90\%ile: $\mathrm{OR}=2.96,95 \% \mathrm{CI}=1.51-5.79$ ), and in the highest category of career-cumulative nickel/chromium-VI combined exposure (>90\%ile: $\mathrm{OR}=1.51,95 \% \mathrm{CI}=1.01-2.26$ ). These relationships weakened in analyses restricted to blue collar workers but interpretations remained unchanged.
Conclusions These results support causal links between exposure to known lung carcinogens and laryngeal cancer. Measures to reduce such exposures in workplaces could decrease the risk of both laryngeal and lung cancers.

\section{O6B.5 VARIATION IN NORDIC WORK-RELATED CANCER RISKS AFTER ADJUSTMENT FOR ALCOHOL AND TOBACCO}

${ }^{1}$ Kristina Kjaerheim*, ${ }^{1}$ Tor Haldorsen, ${ }^{2}$ Elsebeth Lynge, ${ }^{1}$ Jan Ivar Martinsen, ${ }^{3}$ Eero Pukkala, ${ }^{1}$ Elisabete Weiderpass, ${ }^{1} \mathrm{Tom} \mathrm{Kr}$ Grimsrud. ${ }^{1}$ Cancer Registry Of Norway, Oslo, Norge; ${ }^{2}$ Center for Epidemiology and Screening, Institute of Public Health, University of Copenhagen, Copenhagen, Denmark; ${ }^{3}$ Finnish Cancer Registry, Institute for Statistical and Epidemiological Cancer Research, Helsinki, Finland

\subsection{6/OEM-2019-EPI.146}

Background Consumption of alcohol and tobacco strongly increases risk of cancer of the tongue, mouth, pharynx, larynx, and oesophagus, and are established risk factors also for cancer of the liver, colon, and rectum. It is well documented that these habits are unequally distributed among occupational groups. Most occupational cohort studies do not have information on these potentially important confounders, and may therefore be prone to bias.

Aim The aim of the study was to calculate standardized incidence ratios (SIRs) adjusted for alcohol and tobacco by occupation, and to compare to the unadjusted SIRs.

Material and methods The study is based on the Nordic Occupational Cancer (NOCCA) database.

We used confirmatory factor analysis models where the unobserved pattern of alcohol and tobacco consumption were considered a latent common factor, and the potential occupational variation on each cancer type latent site specific factors. Results were used to compute adjusted expected numbers of cancer from the reference rates and to calculate adjusted SIRs for the relevant cancer sites for each occupation.

Results Changes of risk estimates from significantly high to significantly low and vice versa were seen. Among Nordic farmers, unadjusted SIRs for cancer of the mouth and oesophagus were 0.56 (95\% confidence interval [CI] 0.51-0.61) and 0.67 (CI 0.63-0.70), respectively. After adjustment, estimates changed to 1.10 (CI 1.01-1.21) and 1.16 (CI 1.101.22). Unadjusted SIR for pharynx cancer among wood workers was 0.83 (CI 0.75-0.91), adjusted SIR was 1.14 (CI 1.031.25). For larynx cancer, results in the opposite direction were seen: unadjusted SIR for economically inactive was 1.38 (CI 1.31-1.46) while the adjusted SIR was 0.91 (CI 0.86-0.96).

Conclusion Adjustment for the latent indicators of alcohol and tobacco consumption changed risk estimates for several occupations, and may guide in the identification of true risk factors and preventive strategies.

\section{O6B.6 OCCUPATIONAL RADIATION EXPOSURE AND THE RISK OF CANCER AND CARDIOVASCULAR DISEASES AMONG MEDICAL RADIATION WORKERS}

Won Jin Lee*, Eun Shil Cha, Seulki Ko. Korea University College of Medicine, Seoul, South Korea

10.1136/OEM-2019-EPI.147

Background Medical radiation workers occupy the largest group of radiation workers and the numbers are rapidly 
increasing worldwide. This presentation purposes to report findings from the Korean medical radiation workers' study.

Methods Data on all diagnostic medical radiation workers enrolled at the national dose registry between 1996 and 2011 $(n=94,396)$ were merged with the death and cancer incidence data with coverage through the end of 2015. We reconstructed historical radiation doses and estimated organ-specific doses for all workers. The cancer risks were calculated using standardized mortality ratios (SMRs), standardized incidence ratios (SIRs), excess relative risk (ERR), and lifetime attributable risk (LAR). For radiologic technologists $(n=12,906)$, we conducted a survey and merged the data with the national health insurance data of 2006-2016 for investigation of non-cancer diseases.

Results The mean cumulative badge doses for all workers were $10.6 \mathrm{mSv}$ (men) and $2.7 \mathrm{mSv}$ (women). Medical radiation workers have more favorable mortality than in general population for all causes of death among men $(S M R=0.45)$ and women $(\mathrm{SMR}=0.49)$. Based on 2192 of primary cancer cases, the SIR for all cancers significantly decreased in men $(\mathrm{SIR}=0.88)$ and increased in women $(\mathrm{SIR}=1.10)$. However, there were no significant ERRs of all cancer incidences in both men and women. LARs for all cancer combined ranged from 9 to 402 per 100000 varied by sex and job title. Among radiologic technologists, the odds ratios for cardiovascular diseases showed a significant increasing trend with colon doses (trend $\mathrm{p}=0.024$ ) after adjusting for potential risk factors.

Conclusions Our findings provide some evidence of occupation radiation exposure and its health effects among medical radiation workers. The risks were generally small but not acceptable at high risk groups. Continuous monitoring and further followup is warranted to optimize the work practices for the protection of potential health risks in medical radiation workers.

\section{O6B.7 ARISTOLOCHIC ACID AND THE RISK OF CANCERS IN PATIENTS WITH DIABETES}

${ }^{1}$ Chi-Jen Chen, ${ }^{2}$ Wei-Che Chiu, ${ }^{3}$ Yao-Hsien Tseng, ${ }^{4}$ Yao-Hsu Yang, ${ }^{1}$ Chien-Mu Lin, ${ }^{5} H$ siaoYu Yang, ${ }^{6}$ Pat Doyle, ${ }^{5}$ Pau-Chung Chen. ${ }^{1}$ Taipei Medical University, Taipei, Taiwan; ${ }^{2}$ Cathay General Hospital, Taipei, Taiwan; ${ }^{3}$ Taichung Veterans General Hospital, Taipei, Taiwan; ${ }^{4}$ Chang Gung Memorial Hospital, Chiayi County, Taiwan; ${ }^{5}$ National Taiwan University, Taipei, Taiwan; ' London School of Hygiene and Tropical Medicine, London, UK

\subsection{6/OEM-2019-EPI.148}

Background The purpose of this study was to investigate the association between the use of herbal medicine containing aristolochic acid (AA) and the risk of cancers among patients with diabetes.

Methods We conducted a population-based cohort study on patients older than 18 years who had a diagnosis of diabetes (ICD-9 codes 250) between January 1, 1997 and December 31, 2010. To ensure comparability, we included only patients with diabetes who had visited traditional Chinese medicine clinics between January 1, 1997 and one year before the diagnosis of cancer or the censor dates. The use of herbal medicine containing AA was identified from January 1, 1997 to October 31, 2003 (the ban of herbs containing AA in November 2003). Each patient was individually tracked to identify incident cases of cancer (140-208) between January 1, 1999 and December 31, 2013.

Findings A total of 430377 male and 431956 female patients with diabetes were identified by using the National Health Insurance Research Database in Taiwan. There were 37554 and 31535 cancers during the follow-up period. AA use increased the risks of incident liver (155.0), kidney (189.0), pelvis and ureter (189.1, 189.2), and bladder (188) cancer in male patients with diabetes in a dose-dependent relationship. Similarly, there were increased risks of incident liver, pelvis and ureter, and bladder cancer in female diabetic patients in a dose-dependent manner.

Interpretation Our study suggests that AA exposure plays an important role in the carcinogenesis of liver, kidney, pelvis, ureter, and bladder cancers in patients with diabetes.

\section{Cardiovascular Disease}

\section{C.1 ISCHAEMIC HEART DISEASE AND OCCUPATION: A LINKAGE BETWEEN TWO NEW ZEALAND SURVEYS AND THE INTEGRATED DATA INFRASTRUCTURE}

${ }^{1} \mathrm{M}$ Corbin*, ${ }^{1} \mathrm{~L}$ Barnes, ${ }^{1} \mathrm{HJ}$ Denison, ${ }^{1} \mathrm{~A}$ Eng, ${ }^{1} \mathrm{~A}$ Mannetje, ${ }^{1} \mathrm{D}$ Mclean, ${ }^{1} \mathrm{~L}$ EllisonLoschmann, ${ }^{4} \mathrm{R}$ Jackson, ${ }^{1} \mathrm{~J}$ Douwes. ${ }^{1}$ Centre for Public Health Research, Massey University, Wellington, New Zealand; ${ }^{2}$ Institute of Fundamental Sciences - Statistics, College of Sciences, Massey University, Palmerston North, New Zealand; ${ }^{3}$ Statistical Consulting Unit, The Australian National University, Acton Australian Capital Territory, Australia; ${ }^{4}$ Section of Epidemiology and Biostatistics, School of Population Health, Faculty of Medical and Health Sciences, The University of Auckland, Auckland, New Zealand

\subsection{6/OEM-2019-EPI.149}

Background Associations between ischaemic heart disease (IHD) and occupation are poorly understood. We linked two previously conducted New Zealand workforce surveys with routinely collected health data to assess occupational risk factors of IHD in New Zealand.

Methods Two probability-based sample surveys of the general New Zealand adult population $(2004-2006$; $n=3003)$ and of the New Zealand indigenous peoples (Māori; 2009-2010; $\mathrm{n}=2107$ ), for which occupational history was collected, were linked with health data up to the end of 2017 using Statistics New Zealand individual-level microdata. Incident IHD events were identified using hospitalisations, prescriptions and deaths. The odds ratios associated with ever being employed in occupational groups were estimated by logistic regression adjusting for age, smoking and socio-economic status separately for males and females in each cohort.

Results A total of 282 IHD cases were identified in both surveys. Statistically significant elevated IHD risks were observed for male clerks in the general survey $(\mathrm{OR}=1.60,95 \%$ $\mathrm{CI}=1.02-2.49)$ and agriculture and fishery male workers in the Māori survey $(\mathrm{OR}=1.70,95 \% \mathrm{CI}=1.02-2.82)$. Among females, the odds ratios for agriculture and fishery workers were $1.69(95 \% \mathrm{CI}=0.81-3.51)$ and $1.49(95 \% \mathrm{CI}=0.81-2.75)$ in the general survey and the Māori survey, respectively. A statistically significant increased risk was observed for female plant and machine operators and assemblers in the Māori survey $(\mathrm{OR}=1.87,95 \% \mathrm{CI}=1.05-3.31)$. In the general survey, male plant and machine operators and assemblers had an odds ratio of $1.26(95 \% \mathrm{CI}=0.81-1.95)$. We also identified borderline increased odds ratios for trades workers among males in the general survey $(\mathrm{OR}=1.39,95 \% \mathrm{CI}=0.92-2.12$, $\mathrm{p}$-value $=0.12$ ) and among females in the Māori survey $(\mathrm{OR}=2.26,95 \% \mathrm{CI}=0.98-5.21$, p-value $=0.06)$.

Conclusion This study identified associations between several occupational groups and IHD in the New Zealand population. Further analyses will be conducted to assess specific occupational exposures associated with IHD risk. 